\section{Vorgehen bei Patienten mit Teratom mit maligner Transformation}

Giannatempo P et al. Treatment and Clinical Outcomes of Patients with Teratoma with Somatic-Type Malignant Transformation: An International Collaboration. J Urol 2016; 196: 95 100

Teratome mit maligner Transformation somatischer Zellen als Teil von Keimzelltumoren sind eine Seltenheit. Sichere Empfehlungen zur Behandlung gibt es bislang nicht, eine internationale Arbeitsgruppe hat sich nun darum bemüht.

Giannatempo et al. haben Daten von insgesamt 320 Patienten mit Teratomen mit maligner Transformation (TMT) zusammengetragen, die zwischen 1981 und 2014 in 1 von 5 Zentren behandelt worden waren. Primär wollten die Forscher wissen, welche klinischen Faktoren für die Prognose des TMT ausschlaggebend waren. In zweiter Linie untersuchten sie die Rolle der Chemotherapie im Gesamtbehandlungskonzept des TMT, und als klinischen Endpunkt berechneten sie die Gesamtüberlebensraten anhand von Kaplan-Meier-Analysen. Bei 287 der 320 Patienten war der Primärtumor gonadal lokalisiert (89,7\%), bei 17 retroperitoneal $(5,3 \%)$ und bei 16 mediastinal (5\%). Die Diagnose des TMT erfolgte bei knapp der Hälfte der Patienten zum gleichen Zeitpunkt wie die des Keimzelltumors ( $n=139,40,6 \%$ ). Histologisch handelte es sich am häufigsten um Zellen eines Adenokarzinoms, gefolgt von Rhabdomyosarkomen, sonstigen Sarkomen, gemischten Histologien und primitiven neuroektodermalen Tumoren. 49 Patienten präsentierten sich primär mit einem klinischen Stadium I, und bei 14 von ihnen erfolgte lediglich eine Orchiektomie. Bei 28 Männern wurde eine retroperitoneale Lymphknotendissektion durchgeführt, und vitale TMT-Zellen wurden bei gut einem Drittel dieser Patienten $(n=10)$ nachgewiesen. Von den restlichen Patienten wiesen 123 ein geringes Risiko für eine Metastasierung auf, 42 ein intermediäres Risiko und 77 ein hohes Risiko nach den Kriterien der International Germ Cell Cancer
Collaborative Group. Die Mehrzahl erhielt eine primär gegen den Keimzelltumor gerichtete Chemotherapie ( $n=159)$, eine Chemotherapie gegen die TMT-Anteile erfolgte nur bei 14 Pateinten. 147 Patienten wurden ausschließlich operiert. Über eine mediane Nachbeobachtungszeit von 25 Monaten kam es bei 167 Patienten zu einem Rezidiv, 104 Patienten starben. Die 5-Jahres-Überlebensrate bei klinischem Stadium I betrug $83,4 \%$ und sank bei geringem, intermediärem bzw. hohem Risiko auf 69,8\%, 49,1\% bzw. 47,9\%. In der multivariaten Analyse erwiesen sich 3 Faktoren als signifikant mit einer guten Prognose verbunden:

- TMT-Histologie ohne Anteile eines primitiven neuroektodermalen Tumors

- gonadaler Primärtumor (vs. extragonadal) und

- geringe Anzahl von ChemotherapieRegimes vor der TMT-Diagnose

Die Art der Chemotherapie selbst hatte dagegen keinen Einfluss auf das Überleben.

\section{FAZIT}

Insgesamt weisen TMT eine deutlich schlechtere Prognose auf als reine Keimzelltumoren im gleichen Stadium, so die Autoren. Dabei scheint eine geringere chemotherapeutische Vorbehandlung - und ein eher chirurgisches Vorgehen - mit einer besseren Prognose verbunden. Das optimale Zytostatikaregime bleibt weiterhin unklar.

\section{Dr. Elke Ruchalla, Bad Dürrheim}

\section{Kommentar}

Das Teratom mit maligner Transformation ist eine der gefährlichsten histologischen Diagnosen bei Keimzelltumoren. Meist tritt diese Transformation unter Chemotherapie auf, sodass die überwiegende Anzahl der davon betroffenen Patienten durch die histologische Untersuchung der Residualtumoren nach postchemotherapeutischer Resektion diagnostiziert wird.
Eine internationale Verbundarbeitsgruppe hat retrospektiv Daten von 320 Patienten zusammengestellt, bei denen ein Teratom mit maligner Transformation diagnostiziert wurde. Nur 49 der Patienten befanden sich aus den genannten Gründen im klinischen Stadium I, in dem die Therapieentscheidung einfach ist: ohne vaskuläre Invasion würde man eine Überwachungsstrategie empfehlen und nur bei fraglichen Lymphknotenvergrößerungen eine primäre retroperitoneale Lymphadenektomie [1]. Bei den metastasierten Patienten nach Residualtumorresektion ist die weitere Therapie von der Operationsqualität abhängig: kann eine R0 Resektion erreicht werden, muss man nicht weiter behandeln. Problematisch sind multipel metastasierte oder inkomplett operierte Patienten mit maligner Transformation. Diese haben eine sehr schlechte Prognose, weil sie grundsätzlich kaum systemisch zu behandeln sind. Da die Form der Transformation von rhabdomyosarkomatoiden über primitiv neuroektodermale Tumore bis hin zu Adenokarzinomen reicht, ist völlig unklar, welche Chemotherapie bei nicht-resektablen Patienten sinnvoll sein könnte. Entsprechend war weder die Art noch die Gabe einer Chemotherapie prädiktiv und die IGCCCG Klassifikation zeigte keine Unterschiede im Gesamtüberleben. Viele dieser maligne transformierten Teratome entwickeln sich sehr spät und weisen dann spezifische molekulare Paramter auf, die für die Chemorefraktärität verantwortlich sind [2]. Wichtig an dieser retrospektiven Auswertung ist der Hinweis auf diese gefährliche Entität der Keimzelltumoren, die meist unter Chemotherapie entsteht und von einem „ungefährlichen“ Teratom nicht zu unterscheiden ist. Die „take-home-message“ des Artikels ist, dass Teratome nach Chemotherapie immer komplett operiert werden müssen und eine Überwachung von Teratomen $>1-2 \mathrm{~cm}$ Transversaldurchmesser sehr gefährlich sein kann. Dies hat vor allem Implikationen auf die Entscheidung, residuelle Tumoren nach Chemotherapie zu operieren oder zu überwachen. Auch wenn die Entität nicht häufig ist, würde man sich bei einem leitlinienkonformen Verlauf der Therapie mit einer partiellen, markernegativen Remission nach Chemotherapie daher 
im Zweifel (Residuum 1-2cm transversal) eher für als gegen eine Residualtumorresektion aussprechen. Wenn eine Rezidivtherapie vorliegt, dann müssen alle Patienten operiert werden, unabhängig davon, wie groß das Residuum nach Rezidivchemotherapie ist, denn hier ist die Entwicklung von maligne transformierten Teratomen besonders groß und ohne Komplettresektion überleben diese Patienten nur selten.

\section{Der Autor}

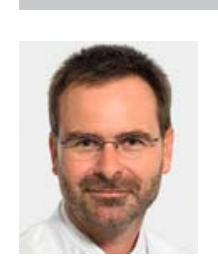

Univ.-Prof. Dr. med. Peter Albers, Direktor der Klinik für Urologie, Universitätsklinikum Düsseldorf

Literatur

[1] Guidelines on Testicular Cancer: 2015 Update. Albers P, Albrecht W, Algaba F, Bokemeyer C, Cohn-Cedermark G, Fizazi K, Horwich A, Laguna MP, Nicolai N, Oldenburg J; European Association of Urology. Eur Urol. 2015: $1054-$ 1068. doi: $10.1016 / j$.eururo.2015.07.044. Epub 2015 Aug 18.

[2] Histopathological and molecular features of late relapses in non-seminomas. Mayer $\mathrm{F}$, Wermann H, Albers P, Stoop H, Gillis AJ, Hartmann JT, Bokemeyer CC, Oosterhuis JW, Looijenga LH, Honecker F. BJU Int. 2011; 107: 936 -943. DO: $10.1111 /$ j.1464 410X.2010.09631.x. Epub 2010 Oct 18. 